\title{
Damage-Free Nanoscale Isotopic Analysis of Biological Materials with Vibrational Electron Spectroscopy
}

Jordan A. Hachtel ${ }^{1, *}$, Jingsong Huang ${ }^{1,2}$, Ilja Popovs ${ }^{3}$, Santa Jansone-Popova ${ }^{3}$, Jong K. Keum ${ }^{1,4}$, Jacek Jakowski $^{1,2}$, Tracy C. Lovejoy ${ }^{5}$, Niklas Dellby ${ }^{5}$, Ondrej L. Krivanek ${ }^{5}$, and Juan Carlos Idrobo ${ }^{1}$

${ }^{1 .}$ Center for Nanophase Materials Sciences, Oak Ridge National Laboratory, Oak Ridge, TN, USA.

2. Computational Sciences and Engineering Division, Oak Ridge National Laboratory, Oak Ridge, TN, USA.

3. Chemical Sciences Division, Oak Ridge National Laboratory, Oak Ridge, TN, USA.

4. Neutron Scattering Division, Oak Ridge National Laboratory, Oak Ridge, TN, USA.

5. Nion R\&D, Kirkland, WA, USA.

*Corresponding author: hachtelja@ornl.gov

Isotopic labeling of biomolecules is a critical aspect for the study of dynamic chemistry in life sciences. Identifying isotopic-labels through conventional mass-spectrometry-based techniques can damage the sample, leading to the loss of valuable structural and chemical information. Recent advances in source monochromation allow electron energy loss spectroscopy (EELS) to be performed in a scanning transmission electron microscope (STEM) with an energy resolution of $\sim 5 \mathrm{meV}$ while maintaining a sub- $\AA$ spatial resolution [1,2]. At these energy levels, subtle variations between the vibrational modes in isotopically labeled samples can be excited and detected with EELS, allowing direct isotopic analysis in the STEM.

Moreover, at these low energies the vibrational EELS can be acquired in 'aloof' mode where the beam is positioned $10 \mathrm{~s}$ of nm away from the sample and the interactions are carried through the evanescent field of fast electrons [3]. As a result, the vibrational response of organic materials can now be accessed with high spatial resolution in the STEM, which is enabled by the ability to collect aloof EELS with a high signal-to-noise ratio and without directly irradiating the sample [4].

Here, we present the vibrational EELS of L-Alanine (L-Ala) both in its naturally occurring ${ }^{12} \mathrm{C}$ isotope and with the carboxylate carbon site labeled with ${ }^{13} \mathrm{C}$. Figure 1a shows aloof vibrational EEL spectra acquired for ${ }^{12} \mathrm{C}$ - and ${ }^{13} \mathrm{C}$-labeled L-Ala. The majority of the spectra overlap between the two L-Ala samples, but the dominant C-O stretch mode peak at $\sim 200 \mathrm{meV}\left(1600 \mathrm{~cm}^{-1}\right)$ is clearly red-shifted due to an increase in the reduced mass of the enriched C-O pair. This small energy shift $(4.8 \pm 0.4 \mathrm{meV})$ is sufficient for the unambiguous identification of isotopically labeled molecules in organic sample [5].

The advantage of this STEM-based method is demonstrated in Figures $1 \mathrm{~b}$ and $1 \mathrm{c}$. By dispersing ${ }^{12} \mathrm{C}$ and ${ }^{13} \mathrm{C}$-labeled L-Ala on the same TEM sample grid, we can leverage aloof EELS to directly identify isotopic labels in real space. By performing a line scan between two clusters of crystalline L-Ala that are separated by a few hundred $\mathrm{nm}$, we can measure the peak position of the $\mathrm{C}-\mathrm{O}$ stretch mode. For the cluster shown on the left of Fig. 1b, the $\mathrm{C}-\mathrm{O}$ stretch mode is centered at $\sim 200.5 \mathrm{meV}$ (the ${ }^{12} \mathrm{C}-\mathrm{O}$ stretch peak) and the cluster on the right is centered at $\sim 195.7 \mathrm{meV}$ (the ${ }^{13} \mathrm{C}-\mathrm{O}$ stretch peak). While the particles are separated by several hundred $\mathrm{nm}$, it is observed that the signal produced close to the clusters is highly deterministic, indicating that particles that are much closer together could still be resolved. More importantly, the measurement is performed without significantly damaging the samples making it repeatable and applicable to beam-sensitive materials where isotopic labeling plays an important role. 
References:

[1] JA Hachtel, AR Lupini, and JC Idrobo, Sci. Rep. 8 (2018), p. 5637.

[2] OL Krivanek, et al., Ultramicroscopy (In Press) (https://doi.org/10.1016/j.ultramic.2018.12.006)

[3] PA Crozier, Ultramicroscopy 180 (2017), p. 104.

[4] JR Jokisaari, et al., Adv. Mater. 30 (2018), 1802702.

[5] JA Hachtel, et al., Science 363 (2019), p. 525.

[6] Research conducted at ORNL's Center for Nanophase Materials Sciences, which is a U.S.

Department of Energy, Office of Science User Facility, and using instrumentation within ORNL's

Materials Characterization Core provided by UT-Battelle, LLC under Contract No. DE-AC05-

00OR22725 with the U.S. Department of Energy.
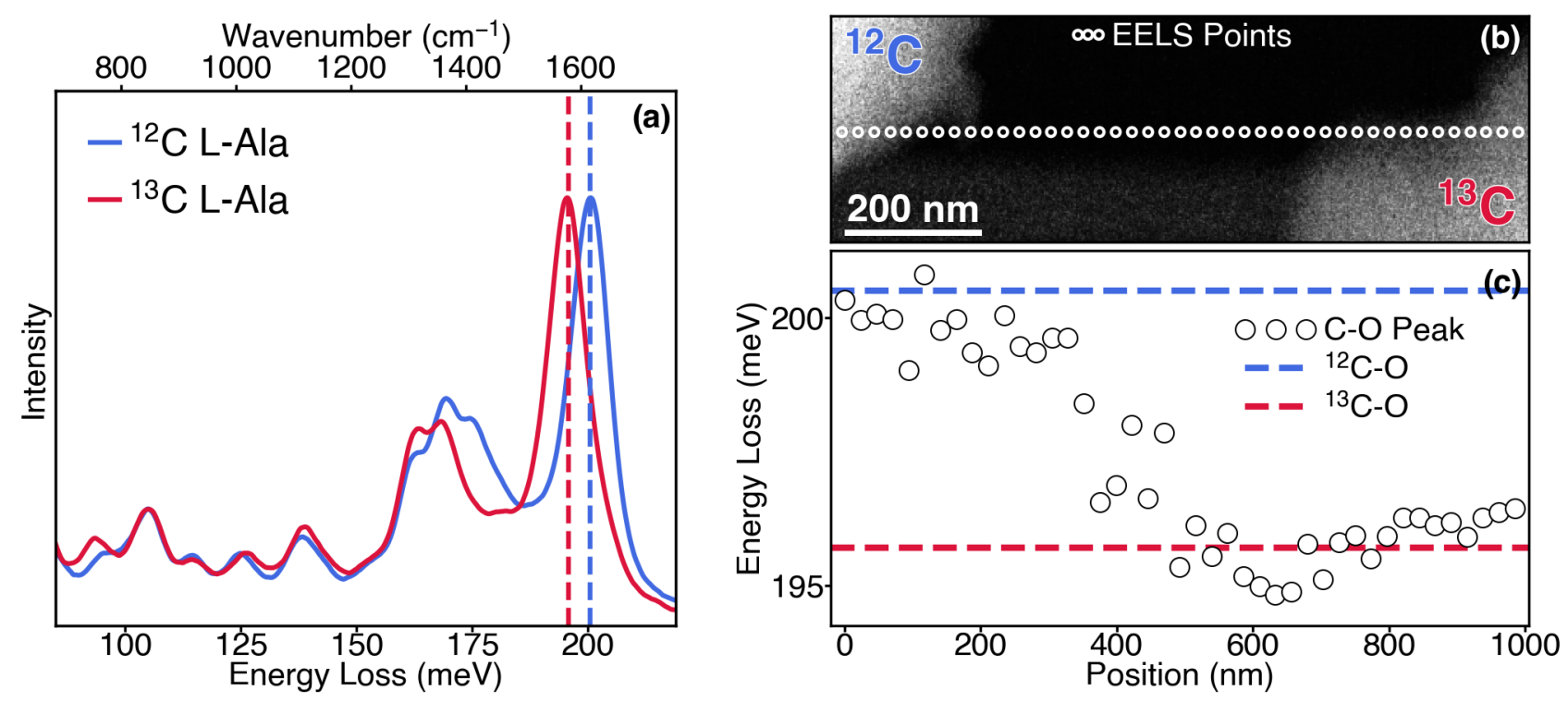

Figure 1. (a) Vibrational EEL spectra for L-Ala, in its naturally occurring ${ }^{12} \mathrm{C}$ form and with ${ }^{13} \mathrm{C}$ isotopic-labeling at carboxylate carbon site. The $\mathrm{C}-\mathrm{O}$ stretch vibrational peak shifts by $\sim 5 \mathrm{meV}$ due to isotopic enrichment. (b) and (c) An EELS line profile between clusters of crystalline L-Ala. Clusters can be identified in real space as either ${ }^{12} \mathrm{C}$ - or ${ }^{13} \mathrm{C}$-labeled through measured peak of $\mathrm{C}$-O stretch mode. 